\title{
EL ÁLGEBRA GEOMÉTRICA COMO MEDIADORA EN LA ENSEÑANZA DE LA FACTORIZACIÓN Y LOS PRODUCTOS NOTABLES
}

\author{
THE GEOMETRIC ALGEBRA AS MEDIATOR IN TEACHING \\ FACTORIZATION AND NOTABLE PRODUCTS
}

Graciela Wagner O. ${ }^{1}$, Alba Marina Giraldo V. ${ }^{2}$,Efraín Alberto Hoyos S. ${ }^{3}$,Heiller Gutiérrez Z. ${ }^{4}$

1. Docente Universidad del Quindío. E-mail: gwagner@uniquindio.edu.co

2. Docente Universidad del Quindío. E-mail: almgiraldo@uniquindio.edu.co

3. Docente Universidad del Quindío. E-mail: eahoyos@uniquindio.edu.co

4. Docente Universidad del Quindío. E-mail: hgutierrez@uniquindio.edu.co

Recibido: 20 de Noviembre de 2014

Aceptado: 10 de Diciembre de 2014

Correspondencia: Graciela Wagner O, Universidad del Quindio, Armenia Quindío Colombia. E-mail: gwagner@uniquindio.edu.co

\section{RESUMEN}

Uno de los retos en la educación matemática es buscar nuevas estrategias que faciliten la comprensión de conceptos matemáticos que se han enseñado de forma mecánica. En esta investigación se orientan los temas de productos notables y la factorización utilizando la estrategia ya conocida como es la geometrización del álgebra, a través de ayudas didácticas de fácil acceso, como son las figuras geométricas. Además, dado que la tecnología está presente en cada momento de nuestra vida, surge la necesidad de incorporar estrategias que permitan al estudiante tener un trabajo dinámico e interactivo; para tal fin se ha implementado el software "geometría de polinomios" como estrategia de enseñanza-aprendizaje de los productos notables y de la factorización de polinomios de segundo grado. Esta estrategia brinda una alternativa didáctica diferente e innovadora que propicia en los estudiantes la consecución de un aprendizaje significativo de estos temas, dejando a un lado la enseñanza tradicional e incorporando dos elementos facilitadores como son la manipulación de material concreto y la utilización de un software, diseñado para el desarrollo de este proyecto. Por otro lado, se cuenta con una cartilla que presenta diferentes actividades para la utilización del material concreto y un manual para el manejo del software.

Palabras claves: Productos, Factorización, Geometrización, Didáctica, Software.

\begin{abstract}
One of the challenges of Mathematics Education is to find new strategies that help understanding mathematical concepts that have being taught in a repetitive way. In this research, we pointed out Notable Products and Factorization using a strategy called the Geometrization of Algebra through easy-access didactic aids such as geometric figures. Also, due to the fact that technology is present in our lives every day, there is the need to incorporate strategies that allow students to perform a dynamic and interactive work; and the best way of doing that is implementing a software called: "Geometry of Polynomials" as a teaching and learning strategy of Notable Products and Factorization of second degree polynomials. Such strategy gives a didactic and innovative alternative that helps students to gain a significant learning of these subjects, leaving behind a traditional way of learning and incorporating two facilitation elements like manipulation of concrete materials and the use of a software specially designed for this project. Lastly, we developed a brochure that shows different activities on how to use the concrete material and a manual for the use of that software.
\end{abstract}

Keywords: Factorization, Geometrization, Didactics, Software. 


\section{INTRODUCCIÓN}

Este estudio de investigación se desarrolla en el campo del pensamiento matemático, en concreto en el desarrollo del pensamiento algebraico, y centra su atención en la comprensión de los conceptos de factorización y productos notables de estudiantes del curso de álgebra del Programa de Licenciatura en Matemáticas y en el espacio académico de cálculo I, del Programa de Ingeniería de Sistemas de la Universidad del Quindío, utilizando el álgebra geométrica como mediadora en el aprendizaje de estos conceptos matemáticos.

Para el estudio y análisis de esta investigación se han utilizado como referentes teóricos los conceptos propios de la aritmética, la geometría y el álgebra, para lo cual se utilizan varios instrumentos y estrategias didácticas como cuestionarios, manipulación de material concreto y el software "Geometría de Polinomios”, para la comprensión de los conceptos matemáticos de factorización y productos notables.

El objetivo de este estudio es el de determinar el nivel de aprendizaje que alcanzan los estudiantes del Programa de Licenciatura en Matemáticas, en la búsqueda de la comprensión de los conceptos de Factorización y Productos Notables, mediante la implementación de estrategias didácticas de enseñanza y de aprendizaje, utilizando la técnica de la Geometrización del Álgebra como un medio para alcanzar un aprendizaje significativo del tema propuesto.

\section{METODOLOGÍA}

Esta investigación realizada en la Universidad del Quindío, es cuantitativa de tipo experimental. Se definen los grupos experimental y el grupo control; tomando como grupo control 30 estudiantes del espacio académico cálculo diferencial del Programa de Ingeniería de Sistemas y como grupo experimental 30 estudiantes del espacio académico de álgebra del Programa de Licenciatura en Matemáticas. Se aplica al grupo control y al grupo experimental el pre-test para conocer los conceptos previos que tienen los estudiantes acerca del tema. Posteriormente, se establecen las condiciones de homogeneidad en los grupos objeto de investigación.

Se desarrolla en el grupo control, el tema de las operaciones básicas, siguiendo el modelo tradicional y se implementa en el grupo experimental la estrategia didáctica: "geometrización del álgebra", usando el material concreto "Álgebra Geométrica" y el software "Geometría de Polinomios";

La estrategia se aplica en tres fases:

En la primera fase se hace el reconocimiento y la manipulación de material didáctico, para identificar geométricamente el concepto de área de cuadrados y rectángulos, lo mismo que la suma de de éstas áreas y su relación con los Productos Notables y la Factorización de polinomios.

Para el desarrollo de los ejercicios se deben tener en cuenta las siguientes figuras:

\begin{tabular}{|l|l|l|}
\hline FIGURA & \multicolumn{1}{|c|}{ DESCRIPCIÓN } & \multicolumn{1}{|c|}{ ÁREA } \\
\hline & Cuadrado de lado arbitrario, [unidad de longitud $1 \mathrm{u}$ (1 unidad)]. & $\mathrm{A}=\mathrm{I}^{*} \mathrm{I}=\mathrm{I}^{2}$ \\
\hline & $\begin{array}{l}\text { Rectángulo de base arbitraria a con la condición que el lado del cuadrado } \\
\text { anterior no sea un múltiplo de la base del rectángulo, pero su altura tiene la } \\
\text { misma dimensión que el lado del cuadrado, esto es } 1 \mathrm{u}\end{array}$ & $\mathrm{A}=\mathbf{a}^{*} 1=\mathbf{a} \mathrm{u}^{2}$ \\
\hline & $\begin{array}{l}\text { Cuadrado de tal forma que su lado, tenga la misma dimensión que la base } \\
\text { del rectángulo anterior }(\mathrm{I}=\mathbf{a})\end{array}$ & $\mathrm{A}=\mathrm{a}^{*} \mathrm{a}=\mathrm{a}^{2} \mathrm{u}^{2}$ \\
\hline & $\begin{array}{l}\text { Rectángulo de base arbitraria } \mathrm{b} \text { menor que } \mathbf{a}, \text { pero a no puede ser un múl- } \\
\text { tiplo de } \mathbf{b}, \mathrm{y} \text { su altura tiene la misma dimensión que el lado del cuadrado } \\
\text { café, esto es } 1 \text { u. }\end{array}$ & $\mathrm{A}=\mathbf{b}^{*} 1=\mathbf{b} \mathrm{u}^{2}$ \\
\hline & $\begin{array}{l}\text { Cuadrado de tal forma que su lado, tenga la misma dimensión que la base } \\
\text { del rectángulo anterior }(\mathrm{I}=\mathbf{b}) .\end{array}$ & $\mathrm{A}=\mathbf{b}^{*} \mathbf{b}=\mathbf{b}^{2} \mathrm{u}^{2}$ \\
\hline & $\begin{array}{l}\text { Rectángulo de base a y de altura } \mathrm{b} \\
\mathrm{N}\end{array}$ & $\mathrm{A}=\mathbf{a}^{*} \mathbf{b}=\mathbf{a b} \mathrm{u}^{2}$ \\
\hline
\end{tabular}


Las actividades propuestas se realizan a través de una guía con información de las características de la estrategia. A continuación se muestran dos ejemplos:

1.Resolver $(a+b)^{2}=(a+b)(a+b)$. En esta multiplicación la base es $\mathrm{a}+\mathrm{b}$; y la altura es $\mathrm{a}+\mathrm{b}$. Como la primera letra de ambos factores es la letra a usamos el cuadrado de lado a (el mayor)
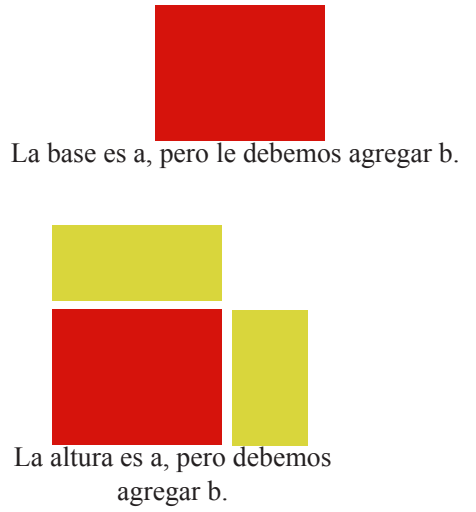

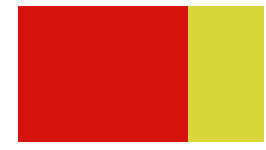

La base es $a+b$

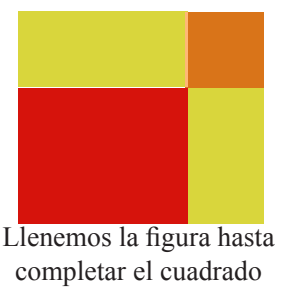

Este último cuadrado esta constituido por las áreas $\mathrm{a}^{2}$ $+2 \mathrm{ab}+\mathrm{b}^{2}$, así la respuesta al ejercicio propuesto es: $(a+b)^{2}=a^{2}+2 a b+b^{2}$.

\section{Factorizar: $a^{2}+2 a$}
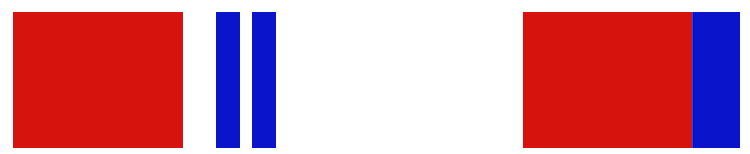

Usamos los rectángulos cuyas áreas sean $\mathrm{a}^{2}$ y $2 \mathrm{a}$. Se construye como una sola figura uniendo las anteriores. Expresamos el área como el producto de sus dimensiones. Altura: a, base $(\mathrm{a}+2)$

Por lo tanto: $\mathrm{a}^{2}+2 \mathrm{a}=\mathrm{a}(\mathrm{a}+2)$

En la segunda fase se hace el reconocimiento y la aplicación del software "Geometría de Polinomios", el cual es un software educativo que permite por medio de fichas, teniendo en cuenta su área y perímetro convertir las expresiones algebraicas en problemas geométricos interesantes, dinámicos y didácticos que propician una estrategia innovadora para la enseñanza de las matemáticas.

Aspectos generales del software "Geometría de Polinomios"

El software creado para esta investigación "Geometría de Polinomios" está programado en la plataforma Delphi complementada con las librerías GLscene ( librerías para desarrollo de aplicaciones en 3D) y utilizando a través de dos módulos (polinomios de grado 2 y polinomios de grado 3) la programación orientada a objetos.

Este software educativo responde particularmente a las posibilidades de entrenamiento de los estudiantes con el fin de que comprendan que los productos notables de polinomios de grado 2 y 3 se pueden representar geométricamente por medio de rectángulos o paralelepípedos respectivamente.

El programa tiene una interfaz amigable que dispone de fichas para cada una de las componentes de la expansión de un polinomio (1,a,axa,b,bxb,c,cxc,axb,axc, bxc, axaxa, axbxc, axaxb, axbxb ,bxbxb, cxcxc). El estudiante debe articular las fichas hasta conformar un rectángulo si el polinomio es de grado 2 o un paralelepípedo si es de grado 3.

Las alternativas que propone la interfaz permiten al estudiante realizar las transformaciones necesarias (traslaciones y rotaciones) para la composición de las gráficas correspondientes a la factorización de los polinomios.

El programa incluye una ayuda que describe el funcionamiento del programa y de todas sus herramientas y además, en formato pdf tiene un manual de instalación y otro de desarrollo de actividades.

Requiere mínimo para su funcionamiento Sistema operativo Windows XP con memoria RAM 512 MB, Procesador Intel Pentium III.

El entorno del software es el siguiente:

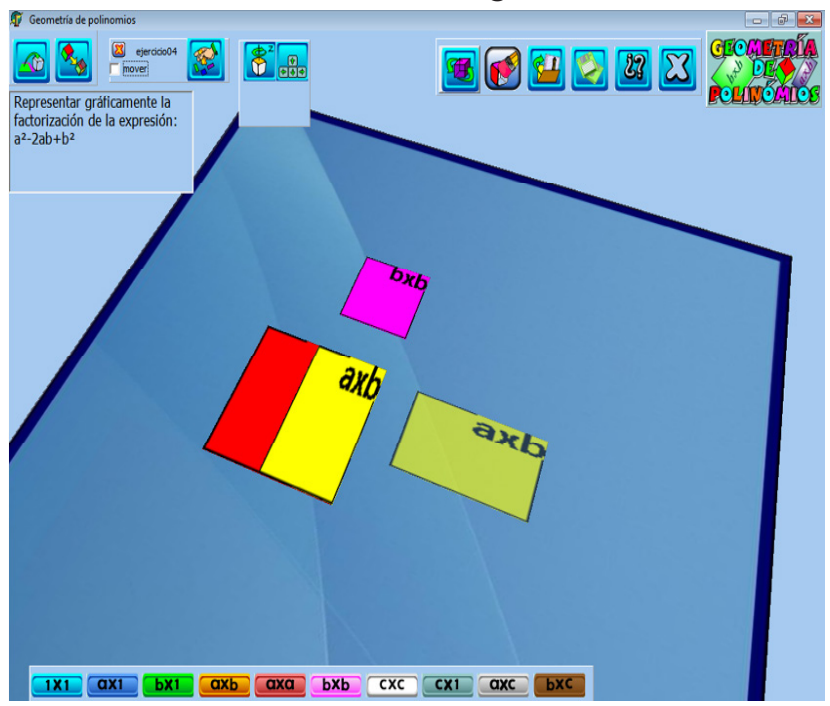

En la tercera fase se hace el análisis estadístico de los resultados obtenidos. 


\section{RESULTADOS Y DISCUSIÓN}

A continuación se presentan los estadísticos utilizados en las pruebas

\begin{tabular}{|c|c|c|}
\hline MEDIA ARITMÉTICA & PRE-TEST & POS-TEST \\
\hline GRUPO CONTROL & 2,42 & 2,91 \\
\hline GRUPO EXPERIMENTAL & 2,71 & 3,39 \\
\hline MEDIANA & PRE-TEST & POS-TEST \\
\hline GRUPO CONTROL & 2,3 & 3,1 \\
\hline GRUPO EXPERIMENTAL & 2,9 & 3,6 \\
\hline \multicolumn{3}{|c|}{$\begin{array}{l}\text { RESULTADOS PRUEBA t-STUDENT PARA } \\
\text { COMPARAR LAS MEDIAS }\end{array}$} \\
\hline $\begin{array}{l}\text { PRE-TEST EXPERIMENTAL- } \\
\text { PRE-TEST CONTROL }\end{array}$ & 0.1827 & GNIFICATIVO \\
\hline $\begin{array}{l}\text { POS-TEST EXPERIMENTAL- } \\
\text { POS-TEST CONTROL }\end{array}$ & 0,0472 & NIFICATIVO \\
\hline
\end{tabular}

\section{PRUEBA DE HOMOGENEIDAD}

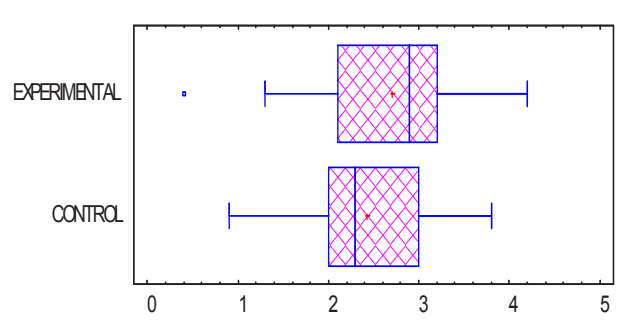

GRÁFICA No.1 ANÁLISIS DE LOS RESULTADOS DE LOS PRE-TEST

En el diagrama de cajas se observa la homogeneidad de los resultados. E1 50\% de los estudiantes del grupo control obtuvo notas por debajo del 2,3 y 50\% de los estudiantes del grupo experimental obtuvo notas por debajo de 2,9.

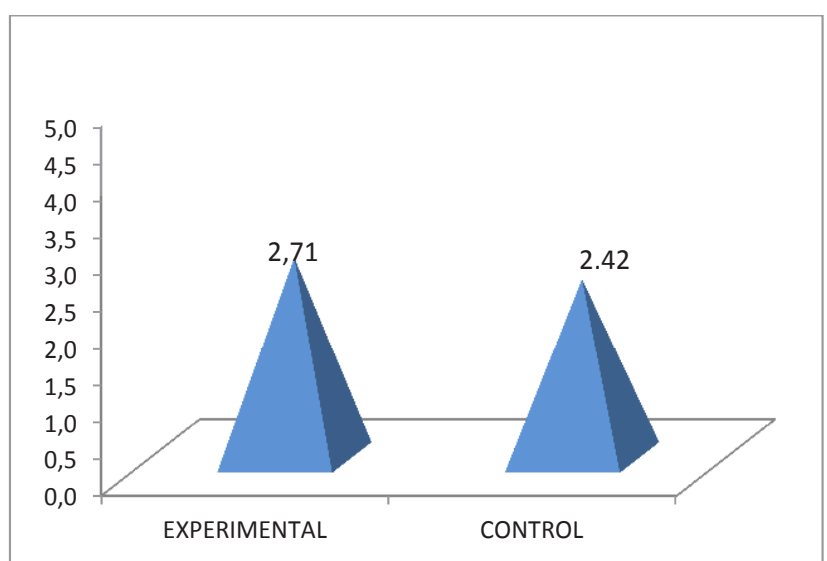

GRÁFICA No.2 COMPARACIÓN PROMEDIOS PRE-TEST
En la prueba t-student realizada para comparar los resultados del pre-test del grupo experimental y del grupo control se observa, que a un nivel de confianza del $95 \%$, no existe diferencia significativa entre los promedios de los grupos, es decir los grupos son homogéneos.

Lo anterior muestra que tanto el grupo control como el grupo experimental obtuvieron resultados muy similares, lo que indica que se encuentran a un mismo nivel de conocimiento frente a la comprensión de la factorización y los productos notables.

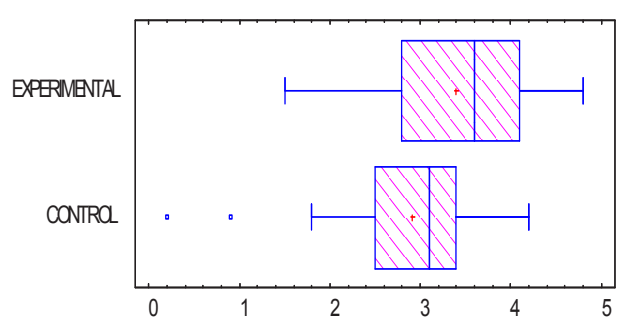

GRÁFICA No.3 COMPARACIÓN DE LOS RESULTADOS DE LOS POS-TEST

En el diagrama de cajas se observa la heterogeneidad de los resultados del pos-test en el grupo control y en el grupo experimental. El 50\% de los estudiantes del grupo control obtuvo notas por debajo del 3.1 y $50 \%$ de los estudiantes del grupo experimental obtuvo notas por debajo del 3.6.

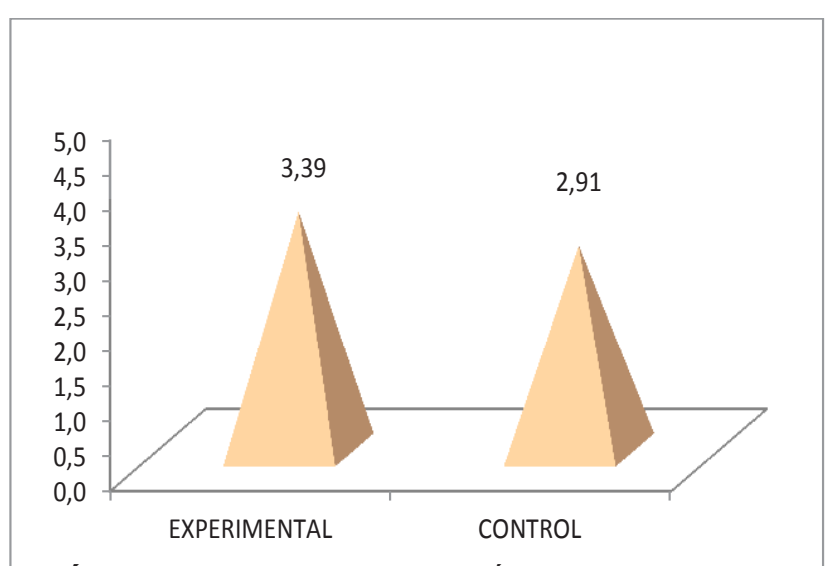

GRÁFICA No.4 COMPARACIÓN PROMEDIOS DEL POS-TEST

En la prueba t-student realizada para comparar los resultados del pos-test del grupo experimental y del grupo control se observa que a un nivel de confianza del 95\%, existe diferencia significativa entre los promedios de los grupos. 


\section{DISCUSIÓN}

Para el desarrollo de esta investigación se tiene en cuenta la estrategia didáctica la Geometrización del Álgebra, en la que son necesarios algunos conocimientos previos, tales como: el área y el perímetro de cuadrados y rectángulos, término algebraico, expresión algebraica, operaciones con expresiones algebraicas.

La implementación de la estrategia didáctica se desarrolla a través de ayudas didácticas de fácil acceso, como el material didáctico "Algebra Geométrica", con el cual se busca que el estudiante visualice el concepto de la factorización mediante la manipulación de material concreto, con el fin de que realice, reconozca y describa patrones, realice analogías para que empiece a construir los conceptos.

En la estrategia los estudiantes deben construir áreas de figuras rectangulares, de manera puedan pensar y razonar en cómo dividirlas de tal forma que al adjuntarlas siempre formen un rectángulo o un cuadrado. Esto permite el desarrollo de cierta creatividad para resolver los ejercicios.

Finalmente se trabaja con el software "Geometría de Polinomios" como estrategia de enseñanza-aprendizaje de los productos notables y de la factorización de polinomios de segundo grado.

El uso de estrategias didácticas sirve para propiciar en los estudiantes mayor interés por el aprendizaje de la factorización y los productos notables; además, se observa un cambio en su forma de razonar, analizar y utilizar el concepto de la factorización y los productos notables en el contexto, obteniendo un aprendizaje mas significativo y no mecánico o repetitivo.

Con los estudiantes del grupo control se trabaja el concepto de factorización y los productos notables utilizando la estrategia didáctica tradicional.

Por ello, en el grupo control, se orientan los temas de la factorización y los productos notables siguiendo los siguientes pasos:

1. Definición de productos notables.

2. Explicación de algunos ejemplos.

3. Se dieron a conocer las fórmulas de produc tos notables y casos de factorización.

4. Desarrollo de ejercicios en clase.

5. Además se realizaron actividades extra clase como: ejercicios y talleres, con el fin de que los estudiantes profundicen en los conceptos desarrollados.

Como se observó en el análisis estadístico, los estudiantes del grupo control tuvieron avances con respecto a su desempeño el cual se evidencia en los resultados del pre-test y pos-test, pero no en la misma medida que en el grupo experimental.

Los resultados alcanzados durante la aplicación de la estrategia didáctica del Algebra Geométrica como mediadora en la enseñanza de la factorización y los productos notables en los estudiantes del grupo experimental, son significativos puesto que los estudiantes mostraron un gran avance en la apropiación de los conceptos.

\section{CONCLUSIONES}

Mediante el uso del material concreto "Álgebra Geométrica" y del software interactivo: "Geometría de Polinomios", se incrementa el interés en los estudiantes y la disposición para el aprendizaje, pues a partir del análisis y de los resultados, se evidencia que los estudiantes logran un mayor nivel de comprensión, de acuerdo a la forma como se enfrentan a las diferentes actividades propuestas. Con el uso de estrategias didácticas se facilita la apropiación de conceptos matemáticos y el desarrollo de habilidades intelectuales superiores (explorar, conjeturar, razonar, reflexionar y comunicar matemáticamente). Además, la manipulación de objetos concretos, lo mismo que el uso de la tecnología, permiten desarrollar en los estudiantes un pensamiento matemático más efectivo y un mayor nivel de apropiación de los conceptos en los estudiantes del grupo experimental, lo cual se comprobó al comparar los resultados del pre-test y pos-test aplicados en ambos grupos.

Los estudiantes muestran mayor interés por el aprendizaje, cuando el docente, además de conocer el tema a profundidad, utiliza estrategias de enseñanza que le permiten hacer análisis a priori y a posteriori de los avances y dificultades de los estudiantes antes, durante y después del aprendizaje del concepto matemático.

Dado que en la actualidad, la tecnología juega un papel tan importante en todos los niveles, es necesario utilizar recursos tecnológicos como ayuda didáctica para fomentar el interés y la apropiación del conoci- 
miento; por lo que se sugiere a los docentes de matemáticas utilizar las estrategias desarrolladas en el presente trabajo para dejar atrás el modelo tradicional y a través de éste desarrollar sus habilidades y gusto por las matemáticas.

\section{BIBLIOGRAFÍA}

- Ausubel, N. (1983). Psicología educativa: Un punto de vista cognoscitivo. $2^{\circ}$ ed. México: Trillas.

- Ausubel, D. (1986). Psicología Educativa. Un enfoque cognoscitivo. Segunda Edición. México: Trillas

- Camargo, L., et al. (2003). Alfa 9 con estándares. Bogotá: Norma.

- Duval, R. (1999). Semiosis y pensamiento humano. Traducción de Myriam Vega. Colombia: Peter Lang.

- Godino, J., et al. (2004). Didáctica de las matemáticas para docentes. Proyecto Edumat- Docentes. Universidad de Granada.

- Godino J. (2003). Razonamiento algebraico y su didáctica para maestros. Universidad de Granada.

- Murillo, P. (2000). ¿Qué es el aprendizaje significativo y cuál es su importancia en el aprendizaje de la matemática? http://www.utp.ac.pa./articulos/aprendizaje significativo.htm. 